\title{
On Kropina metrics
}

\author{
H. G. Nagaraja
}

Received: 11 March 2012 / Accepted: 20 December 2012

(C) African Mathematical Union and Springer-Verlag Berlin Heidelberg 2013

\begin{abstract}
In this paper we study the curvature properties of Kropina metric. We find expressions for Riemann curvature and Ricci curvature of a Kropina metric when the 1-form $\beta$ is a Killing form of constant length. We give a characterization of projectively flat Kropina metric and Kropina metric with isotropic $S$-curvature.
\end{abstract}

Keywords S-curvature $\cdot$ Kropina metric $\cdot$ Projectively flat $\cdot$ Ricci curvature $\cdot$ Riemann curvature.

Mathematics Subject Classification (2010) $53 \mathrm{~B} 40 \cdot 53 \mathrm{C} 60$.

\section{Introduction}

A Finsler metric $L(x, y)$ on an n-dimensional manifold $M^{n}$ is called an $(\alpha, \beta)$-metric [3] $L(\alpha, \beta)$ if $L$ is positively homogeneous function of $\alpha$ and $\beta$ of degree one, where $\alpha^{2}=$ $a_{i j}(x) y^{i} y^{j}$ is a Riemannian metric and $\beta=b_{i}(x) y^{i}$ is a 1 -form on $M^{n}$. Kropina metrics are special $(\alpha, \beta)$ metrics defined in the form $F=\frac{\alpha^{2}}{\beta}$. They form an important class of Finsler metrics investigated by Kropina [2] in 1961. Since then many authors have investigated the geometric properties of Kropina metrics. There are several interesting curvatures in Finsler geometry, among them two important and interesting curvatures are Riemann curvature and the Ricci curvature. The Ricci curvature plays an important role in the geometry of Finsler manifolds and is defined as the trace of the Riemannian curvature on each tangent space. Shen [4] introduced the notion of $S$ - curvature, a non-Riemannian quantity which measures the rate of change of the volume form of a Finsler space along the geodesics. The S-curvature

This work is supported by CSIR, Govt. of India under the grant 25(0179)/10/EMR-II.

H. G. Nagaraja $(\varangle)$

Department of Mathematics, Bangalore University, Central College Campus,

Bangalore 577451, Karnataka, India

e-mail: hgnraj@yahoo.com 
vanishes for Berwald metrics including Riemannian metric. In this paper we give an explicit formula of the Riemannian curvature and the Ricci curvature of a Kropina metric where $\beta$ is a Killing 1-form of constant length. We prove the conditions for a Kropina metric to have isotropic $S$-curvature and to be projectively flat.

\section{Preliminaries}

Let $F=\frac{\alpha^{2}}{\beta}$ be a Kropina metric [2], where $\alpha^{2}=a_{i j}(x) y^{i} y^{j}$ is a Riemannian metric and $\beta=b_{i}(x) y^{i}$ is a 1 -form on an $n$-dimensional manifold $M^{n}$. Let $F^{n}=\left(M^{n}, F\right)$ be the Finsler space equipped with the Kropina metric $F$. Then the space $R^{n}=\left(M^{n}, \alpha\right)$ is called the associated Riemannian space with $F^{n}=\left(M^{n}, L(\alpha, \beta)\right)$. The covariant differentiation with respect to the Levi Civita connection $\gamma_{j k}^{i}(x)$ of $R^{n}$ is denoted by (:). We put $\left(a^{i j}\right)=\left(a_{i j}\right)^{-1}$ and we use the symbols as follows: $[1,6]$

$$
\begin{aligned}
r_{i j}=\frac{1}{2}\left(b_{i ; j}+b_{j ; i}\right), s_{i j}=\frac{1}{2}\left(b_{i ; j}-b_{j ; i}\right), s_{j}^{i}=a^{i r} s_{r j}, s_{j}=b_{r} s_{j}^{r}, b^{i} & =a^{i r} b_{r} a n d b^{2} \\
& =a^{r s} b_{r} b_{s} .
\end{aligned}
$$

Let $F=\alpha \phi(s), s=\frac{\beta}{\alpha}$. For a Kropina metric $F$, we have

$$
\phi(s)=\frac{1}{s} .
$$

The spray coefficients $G^{i}$ of $F$ are given by [1]:

$$
G^{i}=G_{\alpha}^{i}+\alpha Q s_{0}^{i}+\chi\left(-2 \alpha Q s_{0}+r_{00}\right) \frac{y^{i}}{\alpha}+\Psi\left(-2 \alpha Q s_{0}+r_{00}\right)\left\{b^{i}-s \frac{y^{i}}{\alpha}\right\},
$$

where

$$
\begin{aligned}
& Q:=\frac{\phi^{\prime}}{\phi-s \phi^{\prime}}, \\
& \chi:=\frac{\left(\phi-s \phi^{\prime}\right) \phi^{\prime}}{2 \phi\left(\left(\phi-s \phi^{\prime}\right)+\left(b^{2}-s^{2}\right) \phi^{\prime \prime}\right)}, \\
& \Psi:=\frac{\phi^{\prime \prime}}{2\left(\left(\phi-s \phi^{\prime}\right)+\left(b^{2}-s^{2}\right) \phi^{\prime \prime}\right)} .
\end{aligned}
$$

The Riemann curvature is a family of linear maps

$$
R_{y}=R_{k}^{i} \frac{\partial}{\partial x^{i}} \otimes d x^{k}: T_{x} M^{n} \longrightarrow T_{x} M^{n},
$$

defined by

$$
R_{k}^{i}=2 \frac{\partial G^{i}}{\partial x^{k}}-y^{j} \frac{\partial^{2} G^{i}}{\partial x^{j} \partial y^{k}}+2 G^{j} \frac{\partial^{2} G^{i}}{\partial y^{j} \partial y^{k}}-\frac{\partial G^{i}}{\partial y^{j}} \frac{\partial G^{i}}{\partial y^{k}} .
$$

The Ricci curvature and Ricci scalar are defined by

$$
\text { Ric }=R_{i}^{i}, \quad R=\frac{1}{n-1} \text { Ric. }
$$


The S-curvature of a Finsler space $F^{n}=\left(M^{n}, F\right)$ is a scalar function $S: T M^{n} \longrightarrow R$ defined by [4]

$$
S=\frac{\partial G^{m}}{\partial y^{m}}-y^{m} \frac{\partial\left(\ln \sigma_{F}\right)}{\partial x^{m}},
$$

where $\sigma_{F}=\frac{\operatorname{Vol}\left(B^{n}\right)}{\operatorname{Vol}\left\{\left(y^{i}\right) \in R^{n} \mid F\left(x,\left.y^{i} \frac{\partial}{\partial x^{i}}\right|_{x}\right)<1\right\}}$.

A Finsler space $F^{n}=\left(M^{n}, F\right)$ is said to have isotropic $S$-curvature if there exists a smooth function $c=c(x)$ on $M^{n}$ such that $S=(n+1) c F$.

The $E$-curvature $E$ of a Finsler space $F^{n}=\left(M^{n}, F\right)$ is a scalar function $E: T_{P} M^{n} \times$ $T_{P} M^{n} \longrightarrow R$ defined by

$$
E_{i j}=\frac{1}{2} S_{y^{i} y^{j}}
$$

A Finsler space $F^{n}=\left(M^{n}, F\right)$ is said to have isotropic $E$-curvature if there exists a smooth function $c=c(x)$ on $M^{n}$ such that $E=(n+1) c F_{y^{i} y^{j}}$.

\section{Riemann curvature and Ricci-curvature of a Kropina metric}

Let $F=\frac{\alpha^{2}}{\beta}$ be a Kropina metric on an $n$-dimensional manifold $M^{n}$. From (2.2), the geodesic coefficients $G^{i}$ of $F$ are related to the coefficients ${ }^{\alpha} G^{i}$ of $\alpha$ by

$$
G^{i}={ }^{\alpha} G^{i}+P y^{i}+Q^{i}
$$

where

$$
\begin{aligned}
P & =-\frac{1}{b^{2}}\left(s_{0}+F^{-1} r_{00}\right), \\
Q^{i} & =-\frac{1}{2}\left[F s_{0}^{i}-\frac{1}{b^{2}}\left(F s_{0}+r_{00}\right) b^{i}\right] .
\end{aligned}
$$

In this section, we assume that $\beta$ is a Killing form of constant length. i.e., $\beta$ satisfies

$$
r_{i j}=0, \quad \text { and } \quad b^{j} b_{j: k}=0 .
$$

(3.4) implies that

$$
s_{i j}=b_{i: j}, \quad s_{j}=b^{i} s_{i j}=0, \quad b^{i} s^{j}{ }_{i}=b^{i} s_{r i} a^{j r}=-b^{i} s_{i r} a^{j r}=0 .
$$

Thus $P=0$ and (3.1) reduces to

$$
G^{i}={ }^{\alpha} G^{i}+Q^{i}
$$

where

$$
Q^{i}=-\frac{1}{2} F s_{0}^{i}
$$

Now from (2.4) and (3.6), we obtain [5]

$$
R^{i}{ }_{k}={ }^{\alpha} R_{k}^{i}+\left\{2 Q_{: k}^{i}-y^{j}\left(Q_{: j}^{i}\right)_{y^{k}}-\left(Q^{i}\right)_{y^{j}}\left(Q^{j}\right)_{y^{k}}+2 Q^{j}\left(Q^{i}\right)_{y^{j} y^{k}}\right\},
$$

where $\left(Q^{i}\right)_{y^{k}}=\frac{\partial Q^{i}}{\partial y^{k}}$. 
Since $\alpha_{: k}=0$ and $y_{: k}=0$, we have $F_{: k}=\left(\frac{\alpha^{2}}{\beta}\right)_{: k}=-\frac{\alpha^{2}}{\beta^{2}} s_{0 k}$ and $F_{y^{k}}=\left(\frac{\alpha^{2}}{\beta}\right)_{y^{k}}=$ $\frac{1}{\beta}\left(2 y_{k}-\frac{\alpha^{2}}{\beta}\right) b_{k}$. Thus from (3.7), we have

$$
\begin{aligned}
Q_{: k}^{i} & =\frac{\alpha^{2}}{2 \beta^{2}} s_{0 k} s_{0}^{i}-\frac{\alpha^{2}}{2 \beta} s_{0: k}^{i}, \\
y^{j}\left(Q_{: j}^{i}\right)_{y^{k}} & =-\frac{1}{2}\left[\frac{2 y_{k}}{\beta}-\frac{\alpha^{2}}{\beta} b_{k}\right] s_{0: 0}^{i}+\frac{\alpha^{2}}{2 \beta^{2}} s_{k 0} s_{0}^{i}-\frac{\alpha^{2}}{2 \beta} s^{i}{ }_{k: 0}, \\
\left(Q^{i}\right)_{y^{j}}\left(Q^{j}\right)_{y^{k}} & =\frac{\alpha^{2}}{2 \beta^{2}} y_{j} s^{j}{ }_{k} s_{0}^{i}+\frac{\alpha^{2}}{4 \beta^{2}} s_{j}^{i} s^{j}{ }_{0}\left(2 y_{k}-\frac{\alpha^{2}}{\beta} b_{k}\right)+\frac{\alpha^{4}}{4 \beta^{2}} s_{j}^{i} s_{k}^{j}, \\
Q^{j}\left(Q^{i}\right)_{y^{j} y^{k}} & =\frac{\alpha^{2}}{4 \beta^{2}}\left[2 s_{k 0} s_{0}^{i}+\left(2 y_{k}-\frac{\alpha^{2}}{\beta} b_{k}\right) s_{0}{ }_{0} s^{i}{ }_{j}\right] .
\end{aligned}
$$

Plugging them into (3.8), we get

$$
\begin{aligned}
& R_{k}^{i}={ }^{\alpha} R_{k}^{i}+\frac{\alpha^{2}}{\beta^{2}} s_{0 k} s_{0}^{i}-\frac{\alpha^{2}}{\beta} s_{0: k}^{i}+\frac{y_{k}}{\beta} s_{0: 0}^{i}-\frac{\alpha^{2}}{2 \beta^{2}} b_{k} s_{0: 0}^{i_{0}}-\frac{\alpha^{2}}{2 \beta^{2}} s_{k 0} s_{0}{ }_{0}+\frac{\alpha^{2}}{2 \beta} s_{k: 0}^{i} \\
& -\frac{\alpha^{2}}{2 \beta^{2}} y_{j} s^{j}{ }_{k} s^{i}{ }_{0}-\frac{\alpha^{4}}{4 \beta^{2}} s^{i}{ }_{j} s^{j}{ }_{k}+\frac{\alpha^{2}}{\beta^{2}} s_{k 0} s^{i}{ }_{0}+\frac{\alpha^{2}}{2 \beta^{2}} y_{k} s^{i}{ }_{j} s^{j}{ }_{0}-\frac{\alpha^{4}}{4 \beta^{3}} b_{k} s^{i}{ }_{j} s^{j}{ }_{0} .
\end{aligned}
$$

Since $s_{k 0}=-s_{0 k}, y_{j} s_{k}{ }_{k}=s_{0 k}$, (3.13) can be written as

$$
\begin{aligned}
R_{k}^{i}= & { }^{\alpha} R_{k}^{i}-\frac{\alpha^{2}}{\beta} s_{0: k}^{i}+\frac{y_{k}}{\beta} s_{0: 0}^{i}-\frac{\alpha^{2}}{2 \beta^{2}} b_{k} s_{0: 0}^{i}+\frac{\alpha^{2}}{2 \beta} s^{i}{ }_{k: 0} \\
& +\frac{\alpha^{4}}{2 \beta^{2}} y_{k} s^{i}{ }_{j} s^{j}{ }_{0}-\frac{\alpha^{4}}{4 \beta^{3}} b_{k} s^{i}{ }_{j} s^{j}{ }_{0}-\frac{\alpha^{4}}{4 \beta^{2}} s^{i}{ }_{j} s^{j}{ }_{k} .
\end{aligned}
$$

i.e., (3.14) is in the form

$$
R_{k}^{i}={ }^{\alpha} R_{k}^{i}+\alpha^{2}\left(A+B \alpha^{-2}\right),
$$

where

$$
\begin{aligned}
A & =-\frac{1}{\beta} s_{0: k}^{i}-\frac{1}{2 \beta^{2}} b_{k} s_{0: 0}^{i}+\frac{1}{2 \beta} s^{i}{ }_{k: 0}+\frac{\alpha^{2}}{2 \beta^{2}} y_{k} s^{i}{ }_{j} s^{j}{ }_{0}-\frac{\alpha^{2}}{4 \beta^{3}} b_{k} s^{i}{ }_{j} s^{j}{ }_{0}-\frac{\alpha^{2}}{4 \beta^{2}} s_{j}{ }_{j} s^{j}{ }_{k}, \\
B & =\frac{y_{k}}{\beta} s_{0: 0}^{i} .
\end{aligned}
$$

Taking the trace of $R^{i}{ }_{k}$ in (3.14) and using $y_{k} s^{k}{ }_{0: 0}=0$ and $b_{k} s^{k}{ }_{0: 0}=-s_{k 0} s^{k}$, we obtain

$$
\text { Ric }={ }^{\alpha} \text { Ric }-\frac{\alpha^{2}}{\beta} s_{0: k}^{i}-\frac{\alpha^{4}}{4 \beta^{2}} s^{i}{ }_{j} s^{j}{ }_{k} .
$$

Recently Zhang and Shen [7] obtained the relationship between Ricci curvature Ric of $F$ and ${ }^{\alpha}$ Ric of $\alpha$, which reduces to Eq. (3.15), when $\beta$ is a Killing form of constant length.

It is well known that [7], a Finsler metric $F$ on an $n$-dimensional manifold $M^{n}$ is called an Einstein metric if Ric $=\sigma F^{2}$, where $\sigma=\sigma(x)$ is a scalar function on $M^{n}$.

By (3.15), we immediately obtain the following:

$$
\text { Ric }=c F^{2},
$$


where

$$
c=-\frac{1}{4} s^{j}{ }_{k} s^{k}
$$

if and only if

$$
{ }^{\alpha} \operatorname{Ric}=\frac{1}{\beta} s^{k}{ }_{0: k} \alpha^{2} .
$$

In (3.18), LHS ${ }^{\alpha}$ Ric is quadratic in $y$ and RHS is a rational function. Therefore for $n>2$, there exist a scalar function $\lambda$ such that $s_{0: k}=\lambda \beta$. Hence the equation (3.18) takes the form ${ }^{\alpha}$ Ric $=\lambda \alpha^{2}$. i.e. $\alpha$ is an Einstein metric. Thus we have

Theorem 3.1 A Kropina metric $F=\frac{\alpha^{2}}{\beta}$, where $\beta$ is a Killing 1-form of constant length on an $n$-dimensional manifold $M^{n}(n>2)$ is an Einstein metric if and only if $\alpha$ is also an Einstein metric.

Let us take a local orthonormal frame $\left\{\mathbf{b}_{\mathbf{i}}\right\}$ on $M^{n}$ with respect to $\alpha$ and $e_{i}=\left(y, \mathbf{b}_{\mathbf{i}}\right)_{i=1}^{n}$, a local orthonormal frame on $\Pi^{*} T M^{n}$ determined by $\left\{\mathbf{b}_{\mathbf{i}}\right\}$. Then $\alpha=\sqrt{\sum_{i=1}^{n}\left(y^{i}\right)^{2}}, \beta=\sum b_{i} y^{i}$, $y=y^{i} \mathbf{b}_{\mathbf{i}}$. Equation (3.14) still holds for the coefficients of the Riemann curvature with respect to $\left\{e_{i}\right\}_{i=1}^{n}$. We have

$$
Q^{i}=-\frac{1}{2} F s_{p}^{i} y^{p}=-\frac{1}{2} F b_{i: p} y^{p} .
$$

Therefore (3.14) takes the form

$$
\begin{aligned}
R_{k}^{i}= & { }^{\alpha} R_{k}^{i}-F b_{i: j: k} y^{j}+\left(\frac{y_{k}}{\beta}-\frac{F}{2 \beta} b_{k}\right) b_{i: j: p} y^{j} y^{p}+\frac{1}{2} F b_{i: k: j} y^{j} \\
& +\frac{F}{2 \beta} y_{k} b_{j: p} y^{p} b_{i: r} y^{r}-\frac{F^{2}}{4 \beta} b_{k} b_{i: j} b_{j: p} y^{p}-\frac{F^{2}}{4 \beta} b_{i: j} b_{j: k} .
\end{aligned}
$$

Using the Ricci identity

$$
b_{i: j: k}-b_{i: k: j}=b_{m}{ }^{\alpha} R_{i j k}^{m},
$$

we have [5]:

$$
b_{i: j: k} y^{j}=b_{i: k: j} y^{j}+b_{m}{ }^{\alpha} R_{i j k}^{m} y^{j}
$$

and

$$
\begin{aligned}
b_{i: j: k} y^{j} y^{k} & =-b_{j: i: k} y^{j} y^{k} \\
& =-\left(b_{j: k: i}+b_{m}{ }^{\alpha} R_{i j k}^{m}\right) y^{j} y^{k} \\
& =-b_{m}^{\alpha} R_{i}^{m} .
\end{aligned}
$$

Plugging them into (3.20), we obtain

$$
\begin{aligned}
R_{k}^{i}{ }_{k}= & { }^{\alpha} R_{k}^{i}-F\left(b_{i: k: j}+b_{m}{ }^{\alpha} R_{i}{ }^{m}{ }_{j k}\right) y^{j}-\left(\frac{y_{k}}{\beta}-\frac{F}{2 \beta} b_{k}\right) b_{m}{ }^{\alpha} R_{i}^{m}+\frac{1}{2} F b_{i: k: j} y^{j} \\
& +\frac{F}{2 \beta} y_{k} b_{j: p} y^{p} b_{i: r} y^{r}-\frac{F^{2}}{4 \beta}\left[b_{k} b_{i: j} b_{j: p} y^{p}-b_{i: j} b_{j: k}\right] .
\end{aligned}
$$

Since $\alpha$ is a Riemannian metric,

$$
{ }^{\alpha} R_{i h k}^{h}=-{ }^{\alpha} R_{h i k}^{h}=-\frac{(n-1)}{2}{ }^{\alpha} R_{y^{i} y^{k}} .
$$


Contracting (3.22) with respect to $i$ and $k$ and using (2.5), we obtain an equation for the Ricci curvature Ric of $F$ as follows:

$$
\text { Ric }={ }^{\alpha} R i c-\frac{n-1}{2 \beta} \frac{\alpha^{2}}{\beta} b_{m}{ }^{\alpha} R_{y^{m}} y^{r} y^{r}+\frac{\alpha^{2}}{2 \beta^{2}} b_{m}{ }^{\alpha} R^{m}{ }_{i} b_{i}-\frac{\alpha^{4}}{4 \beta^{2}}\left(b_{i: j}\right)^{2},
$$

where ${ }^{\alpha}$ Ric is the Ricci curvature of $\alpha$.

\section{The S-curvature and projectively flat Kropina metric}

In this section, we focus on the $S$-curvature of a Kropina metric $F=\frac{\alpha^{2}}{\beta}$.

Differentiating (3.1), (3.2) and (3.3) with respect to $y^{i}$, we obtain

$$
\begin{gathered}
G^{m} y^{m}={ }^{\alpha} G_{y^{m}}^{m}+\left(P y_{m}\right)_{y^{m}}+Q_{y^{m}}^{m}, \\
\left(P y^{m}\right)_{y^{m}}=(n+1) P,
\end{gathered}
$$

and

$$
Q_{y^{m}}^{m}=\left(\frac{F}{\beta}-\frac{1}{b^{2}}\right) s_{0}-\frac{1}{b^{2}} r_{0 m} b^{m}-\frac{1}{2} F S_{m}^{m},
$$

where $G^{m}{ }_{y^{m}}=\frac{\partial G^{m}}{\partial y^{m}}$.

Using (4.1), (4.2) and (4.3) in (2.6) we get

$$
\begin{aligned}
S= & { }^{\alpha} G_{y^{m}}^{m}-\frac{1}{b^{2}}\left[(n+2) s_{0}+(n+1) F^{-1} r_{00}+r_{0 m} b^{m}\right] \\
& +\frac{\alpha^{2}}{\beta^{2}} s_{0}-\frac{1}{2} F S^{m}{ }_{m}-y^{m}\left(\ln \sigma_{F}\right)_{x^{m}},
\end{aligned}
$$

where $\left(\ln \sigma_{F}\right)_{x^{m}}=\frac{\partial\left(\ln \sigma_{F}\right)}{\partial x^{m}}$.

We note that [1]

$$
\sigma_{F}(x)=\rho^{n+1} \sigma_{\alpha}(x),
$$

where $\rho=\rho(x)$.

For a Riemannian metric $\alpha$, we have

$$
y^{m}\left(\ln \sigma_{\alpha}\right)_{x^{m}}={ }^{\alpha} G_{y^{m}}^{m} .
$$

Plugging (4.5) and (4.6) in (4.4), we have

$$
\begin{aligned}
S= & -\frac{1}{b^{2}}\left[(n+2) s_{0}+(n+1) F^{-1} r_{00}+r_{0 m} b^{m}\right] \\
& +\frac{\alpha^{2}}{\beta^{2}} s_{0}-\frac{1}{2} F S^{m}{ }_{m}-(n+1) \rho^{-1} \rho_{x^{m}} y^{m} .
\end{aligned}
$$

Suppose $\beta$ is a Killing form (i.e. $\left.r_{i j}=0\right)$ and $S=(n+1) c F$, where $c=-\frac{1}{2(n+1)} S^{m}{ }_{m}$. Then from (4.7), we have

$$
\left((n+2) \beta^{2}-b^{2} \alpha^{2}\right) s_{0}-(n+1) b^{2} \beta^{2} \rho^{-1} \rho_{x^{m}} y^{m}=0 .
$$


The above equation may be expressed as

$$
\left(c_{l m} s_{n}+d_{l m} \rho_{x^{n}}\right) y^{l} y^{m} y^{n}=0,
$$

where

$$
c_{l m}=\left((n+2) b_{l} b_{m}-b^{2} a_{l m}\right),
$$

and

$$
d_{l m}=-(n+1) b^{2} b_{l} b_{m} \rho^{-1} .
$$

Differentiating (4.9) successively with respect to $y^{i}, y^{j}$ and $y^{k}$, we obtain

$$
c_{i j} s_{k}+d_{i j} \rho_{x^{k}}+(i j k)=0,
$$

where $+(i j k)$ denote the cyclic interchange of $i, j, k$ and summation.

Conversely, suppose (4.12) holds. Then multiplying (4.12) by $y^{i} y^{j} y^{k}$, we obtain (4.8). Plugging (4.8) in (4.7), we get $S=(n+1) c F$, where $c=c(x)$, some function of $x$. i.e. $F$ has isotropic $S$-curvature. Thus we have

Proposition 1 The $S$-curvature $S$ of a Kropina metric $F=\frac{\alpha^{2}}{\beta}$ is given by (4.7). If $\beta$ is a Killing form then the following are equivalent:

(1) F has isotropic $S$-curvature.

(2) $c_{i j} s_{k}+d_{i j} \rho_{x^{k}}=0$, where $c_{i j}$ and $d_{i j}$ are given by (4.10) and (4.11).

If $\beta$ is a Killing form of constant length then from (4.7), it follows that $S=(n+1) c F$, where $c=c(x)$, some function of $x$.

Conversely, suppose $F$ has isotropic $S$-curvature, then from (4.8), we have

$$
(n+2) \beta^{2} s_{0}-b^{2} \beta^{2} \rho^{-1} \rho_{x^{m}} y^{m}=b^{2} \alpha^{2} s_{0} .
$$

Comparing the coefficients of $\alpha^{2}$ on both sides, we get $s_{0}=0$. This implies $s_{i}=0$. i.e. $\beta$ is of constant length. Thus we have

Proposition 2 If $F=\frac{\alpha^{2}}{\beta}$ is a Kropina metric and $\beta$ is a Killing form, then the following are equivalent:

(1) $\beta$ is of constant length.

(2) F has isotropic S-curvature.

Combining Propositions 1 and 2, we have the following:

Theorem 4.2 The S-curvature of a Kropina metric is given by (4.7). If $\beta$ is a Killing form then the following are equivalent:

(1) $\beta$ is of constant length.

(2) F has isotropic $S$-curvature.

(3) $c_{i j} s_{k}+d_{i j} \rho_{x^{k}}=0$, where $c_{i j}$ and $d_{i j}$ are given by (4.10) and (4.11).

Next we consider projectively flat Kropina metrics. Let the Kropina metric $F=\frac{\alpha^{2}}{\beta}$ be projectively flat and $\beta$ is a Killing form of constant length. Then $G^{i}=\tilde{P} y^{i}$ and $r_{i j}=0, s_{i}=$ 0 . Therefore (3.1) and (3.2) yield

$$
\begin{gathered}
\tilde{P} y^{i}={ }^{\alpha} G^{i}+Q^{i}, \\
(n+1) \tilde{P} y^{i}={ }^{\alpha} G^{m} y^{m} y^{i}+Q^{m}{ }_{y}^{m} y^{i} .
\end{gathered}
$$


From (4.14) and (4.15), we have

$$
Q^{m}{ }_{y^{m}} y^{i}-(n+1) Q^{i}={ }^{\alpha} G^{m}{ }^{m} y^{i}-{ }^{\alpha} G^{i} .
$$

Using (3.3) and (4.3) in (4.16), we have

$$
\alpha^{2}\left(S^{m}{ }_{m} y^{i}-S_{0}^{i}\right)=2 \beta\left((n+1)^{\alpha} G^{i}-{ }^{\alpha} G^{m} y^{m} y^{i}\right) .
$$

Since $\left((n+1)^{\alpha} G^{i}-^{\alpha} G^{m} y^{m} y^{i}\right)$ is quadratic in $y^{i}$, both sides are identically zero. i.e. $\alpha$ is projectively flat and

$$
S_{m}^{m} y^{i}-S_{0}^{i}=0
$$

Differentiating (4.18) with respect to $y^{i}$ we get $S^{m}{ }_{m}=0$.

Putting this in (4.18), we get $S_{0}^{i}=0$. Therefore $\beta$ is closed.

Conversely suppose $\beta$ is closed and $\alpha$ is projectively flat. Then from (3.1), it follows that $F$ is projectively flat.

Thus we have

Theorem 4.3 A Kropina metric $F=\frac{\alpha^{2}}{\beta}$, where $\beta$ is a Killing form of constant length is projectively flat if and only if $\beta$ is closed and $\alpha$ is projectively flat.

From Theorems 4.2 and 4.3, we have

Theorem 4.4 For a Kropina metric $F=\frac{\alpha^{2}}{\beta}$ with $\beta$ as a Killing form, the following are equivalent:

(1) F has isotropic S-curvature.

(2) $F$ is projectively flat.

(3) $\alpha$ is projectively flat and $\beta$ is closed.

If $\beta$ is a Killing form, then from (4.7) and (2.7), we have

$$
\begin{aligned}
E_{i j}= & \frac{1}{2}\left[\frac{1}{\beta}\left(F_{y^{j}} s_{i}+F_{y^{i}} s_{j}\right)-\frac{1}{\beta^{2}}\left(F_{y^{j}} b_{i}+F_{y^{i}} b_{j}\right) s_{0}+F_{y^{i} y^{j}} \frac{1}{\beta} s_{0}\right. \\
& \left.+F\left(2 \beta^{-3} b_{i} b_{j} s_{0}-\frac{1}{\beta^{2}}\left(b_{i} s_{j}-b_{j} s_{i}\right)\right)-\frac{1}{2} S^{m}{ }_{m} F_{y^{i} y^{j}}\right] .
\end{aligned}
$$

From (4.19), it follows that $E_{i j}=(n+1) c F$, where $c=-\frac{1}{4(n+1)}$ if and only if

$$
\begin{aligned}
& \frac{1}{\beta}\left(F_{y^{j}} s_{i}+F_{y^{i}} s_{j}\right)-\frac{1}{\beta^{2}}\left(F_{y^{j}} b_{i}+F_{y^{i}} b_{j}\right) s_{0}+F\left(2 \beta^{-3} b_{i} b_{j} s_{0}\right. \\
& -\frac{1}{\beta^{2}}\left(b_{i} s_{j}-b_{j} s_{i}\right)+F_{y^{i} y^{j}} \frac{1}{\beta} s_{0}=0 .
\end{aligned}
$$

But (4.20) holds if and only if $s_{0}=0$ or $\beta$ is of constant length. Thus we have

Theorem 4.5 A Kropina metric $F=\frac{\alpha^{2}}{\beta}$ with $\beta$ as a Killing form has isotropic E curvature if and only if $\beta$ is of constant length.

Acknowledgments The author is grateful to the referees for the valuable suggestions and comments towards the improvement of the paper. 


\section{References}

1. Chern, S.S., Shen, Z.: Riemann-Finsler Geometry. World Scientific, Singopore (2005)

2. Kropina, V.K.: On projective two dimensioanl Finsler spaces with special metric. Trudy Sem. Vector Tenzor Anal. 11, 277-292 (1961)

3. Matsumoto, M.: Foundations of Finsler geometry and special Finsler spaces. Kaiseisha Press, Otsu (1986)

4. Shen, Z.: Volume comparision and its applications in Riemann-Finsler geometry. Adv. Math. 128, 306-328 (1997)

5. Shen, Z.: Lectures on Finsler Geometry. World Scientific, Singapore (2001)

6. Shen, Z.: Finsler metrics with $K=0$ and $S=0$. Can. J. Math. 55(1), 112-132 (2003)

7. Zhang, X., Shen, Y.-B.: On Einstein Kropina Metrics. arXiv:1207.1944v1 [math.DG] (2012) 